\section{Society should decide on UK badger cull}

As epidemiologists, we agree with the chief scientist of the UK Department for Environment, Food and Rural Affairs that badger culling is an "evidencebased" policy for controlling bovine tuberculosis (TB) in Britain (Nature http://doi. org/mxd; 2013). We disagree with other, less positive interpretations of that evidence (Nature 490, 317-318; 2012).

The evidence comes from a large-scale, long-term, government project - the Randomised Badger Culling Trial (RBCT). This roughly halved the incidence of TB in cattle herds in the culling area following 4-7 annual badger culls (H. E. Jenkins et al. PLoS ONE 5, e9090; 2010). The reductions in disease were not immediately evident owing to the long incubation period of bovine TB. There were also transient increases in incidence outside the cull areas, attributed to increased badger movements.

The mixed reaction to the RBCT results might reflect the way the data were collated and interpreted. Widely quoted summary statistics from the RBCT include data collected at the start (before any effect was likely) and long after culling had ceased. In our view, this approach is appropriate for testing for statistical significance but is not the best way to quantify effectiveness for a trial.

These analyses were never intended to account for the nonlinear impact of sustained reductions in transmission. For bovine TB in England, where various control measures are already in place, basic epidemiological principles predict that even a small reduction in transmission rate could have large benefits.

All this has confused the debate, and risks compromising the interpretation of future trials of alternative control options, such as badger vaccination.

Planned pilot studies will establish whether badger control is practicable, humane and safe. It is for society to decide whether badgers should be culled at all.

Mark Woolhouse University of Edinburgh, UK. mark.woolhouse@ed.ac.uk James Wood University of Cambridge, UK.

\section{InterAcademy Panel to inform policy}

The InterAcademy Panel (IAP) of the Global Network of Science Academies, co-chaired by two of us (V.t.M. and M.H.), draws on the best international science and is free of vested political and commercial interests. It is in a strong position to identify and inform emerging policy issues.

Many science academies already advise governments on national policy-making and some contribute at a regional level, including the European Academies Science Advisory Council. Bringing together regional networks within the IAP encourages sharing of good practice and generates resources for tackling global issues such as sustainable development.

The IAP is working with the scientific community through its science-education programme and helped to create the Global Young Academy to support the development of early-career scientists. It recently issued a statement on population as part of its aim to address the world's strategic priorities (see go.nature. com/whxzi4).

The immediate challenges for the IAP are to build critical mass, to develop sustained links with policy-makers rather than waiting for a policy crisis, and to ensure accountability with the wider scientific community. Volker ter Meulen, Mohamed Hassan IAP, Trieste, Italy. volker.termeulen@mail.uniwuerzburg.de

Robin Fears German National Academy of Sciences Leopoldina, Halle, Germany.

\section{Nuclear plans add to pressure on Caatinga}

Brazil's government is planning to build a nuclear power plant on the São Francisco River in the semi-arid Caatinga region, which is experiencing a long and severe drought (see R. L. M. Novaes et al. Nature 498, 170; 2013). Now is not the time to put the area's most important water resource under even more pressure.

The river is already heavily used as a water supply and for power generation, irrigation, aquaculture, transport and tourism. The Caatinga region is home to traditional communities whose main livelihoods are fisheries and agriculture. The power plant would displace these communities, resulting in the loss of physical assets and undermining their cultural identity. Water from the reactor cooling system could raise the river's temperature, threatening the survival and balance of ecological networks.

As with the Belo Monte dam, the government is again pushing ahead with a major infrastructure project with seemingly little thought for the social, cultural or environmental consequences.

Erika dos Santos Nunes ${ }^{\star}$ State University of Bahia, Salvador, Brazil.

erika.santosnunes@hotmail.com ${ }^{*}$ On behalf of 4 co-signatories. See go.nature.com/tenkss for full list.

\section{Relaxed laws imperil Australian wildlife}

Policy and legislative changes by Australia's state governments are eroding the vital protection of the country's unique biodiversity.

Reserves are being opened up to ecologically disruptive activities, such as grazing by domestic livestock, logging, mining, recreational hunting and fishing, and commercial development. Protected habitats on private and leasehold land are imperilled too. Queensland and Victoria, for example, are relaxing hard-won laws that limit vegetation clearance on private land, further accelerating the loss of regional biodiversity.

Collectively, these actions increase the pressure on biodiversity conservation in protected areas, many of which are already showing biodiversity loss (for example, the Kakadu National Park in northern Australia). Ecological connectivity is being lost, which will hamper the dispersal of species and their ability to respond to climate-change effects.

Species extinctions are primed to increase. Too many of the country's unique fauna and flora have been wiped out over the past two centuries (see, for example, C. Johnson Australia's Mammal Extinctions; Cambridge Univ. Press, 2006), including the Christmas Island pipistrelle bat (Pipistrellus murrayi) in 2009.

There could be no worse time to weaken reserve protection and relax laws designed to reduce habitat loss.

Euan G. Ritchie ${ }^{\star}$ Deakin University, Burwood, Victoria, Australia. e.ritchie@deakin.edu.au ${ }^{*}$ On behalf of 21 co-signatories. See go.nature.com/xhhtgb for full list.

\section{American football is clear on uncertainty}

Nic Fleming suggests that soccer fans at this month's Confederations Cup in Brazil should recognize that electroniceye verdicts on goal-line accuracy are not always definitive (Nature 497, 537; 2013). By contrast, American football fans are already well aware of the limitations of replays.

In American football, a call is overturned only if there is indisputable visual evidence from the video review that the on-field call was incorrect. If the replay results are ambiguous, the field call stands.

Eric Altschuler New Jersey Medical School, Newark, USA. altschel@umdnj.edu 